\title{
Small and Medium-sized Enterprises (SEMs) Supply Chain Coordinative Innovation Motivations
}

\author{
Ping-Chuan WEN ${ }^{1,{ }^{*}}$, Chen-Min $\mathrm{SHI}^{1, \mathrm{a}}$ \\ ${ }^{1}$ School of Economics and Management, Chongqing University of Posts and Telecommunications, \\ Chongqing, 400065, China \\ ashichenmin.mei@163.com \\ ${ }^{*}$ Corresponding author
}

Keywords: Small and medium-sized enterprises, Coordinative innovation, Motivations.

\begin{abstract}
Small and medium-sized enterprises should take innovation activities if they will get development well, at the same time, making collaboration with other firms is the key for their innovation. This paper discusses several different motivations for SEMs supply chain to make innovative collaboration: (1) the needs of innovation development for SEMs; (2) risks sharing and complementary resources; (3) threats and temptations from innovative small and medium-sized enterprises.

While SEMs accounted for most of the total of enterprises, so their development is related to the economics of society. Making effective measures for SEMs supply chain's innovative collaboration is important.
\end{abstract}

\section{Introduction}

Small and medium-sized enterprises (SEMs) have played important roles on the growth of economics and the increasing employment. However, as the rapid development of globalization, SEMs have faced giant challenges and chances: on one hand, SEMs have to improve their innovation capabilities to adapt to the fierce competition. Because of their flexible system of organization and strong adaptability, SEMs' innovation efficiency are higher than those large corporations[1][2]; On the other hand, with the limited scale and resources, SEMs hardly bear enormous pressure on R\&D capabilities, raising funds and taking risks. In order to effectively represent the innovation advantages of SMEs and to make up for its shortcomings in the innovation process, supply chain collaboration help SEMs maximize the effectiveness of innovation by reducing the innovation risks and increasing the sharing information. Innovation is a key driver to promote the competitiveness of corporates. What's more, supply chain collaboration is an important prerequisite for success.

\section{The needs of Innovation development for SMEs}

The section headings are in boldface capital and lowercase letters. Second level headings are typed as part of the succeeding paragraph (like the subsection heading of this paragraph).

With a variety of roles, SEMs in the supply chain face a complex operating environment (see Fig. 1: SME Supply Chain Structure): First, as a buyer, SEMs purchase raw materials from upstream suppliers. In order to maintain long-term partnerships and to boycott the competition from the large enterprises, SEMs have to enhance the purchase price, which is the main indicator for suppliers to select partners [3]; Second, SEMs will sell processed products to downstream firms. As well, SEMs must satisfy the strict requirements that buyers propose about the quality, price and delivery, etc[4]. 


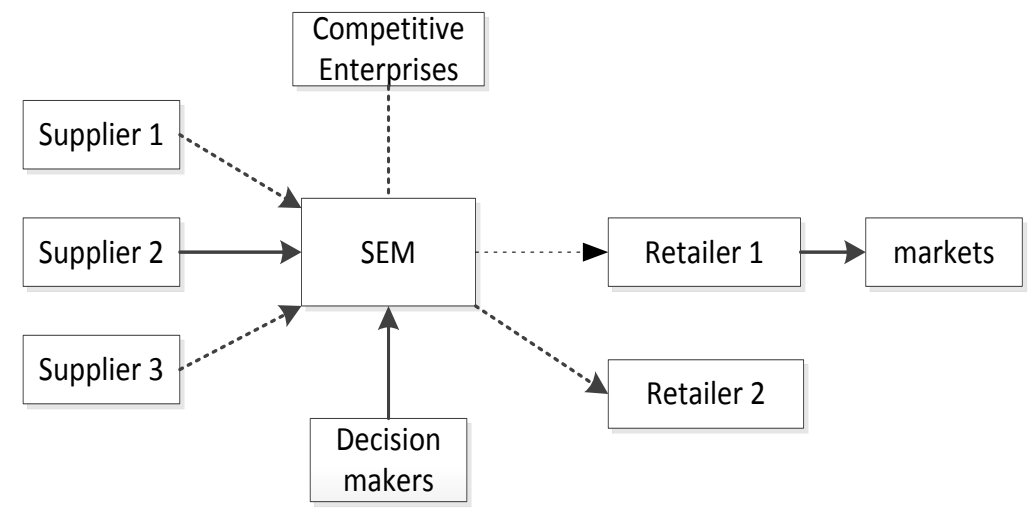

Fig. 1 SME Supply Chain Structure

SEMs in the supply chain not only coordinate the normal operation of the upstream and downstream enterprises, but also wary of the threat of competitive enterprises. Therefore, it's necessary for SEMs to improve their own competitiveness.Innovation helps SEM improve its advantages in some extent. At the same time, because of spillovers effect, other companies in supply chain are also benefited without any action, which is unfair for SEMs. Meanwhile, it will cut down innovation enthusiasm of SEMs.

The development of supply chain complies with" Competition-cooperation- coordination", so supply chain innovation is of Interdependence, rely solely on an independent firm is infeasible. Especially, SEMs hardly assume the responsibility as a result of the limited resources. Therefore, SEMs' innovation must get supports from other companies in the supply chain.

\section{Risks Sharing, Complementary Resources}

As the rapid development of information technology, SEMs are difficult to survive only by relying on unchanged technology and management. Under such circumstances, innovation in the SEMs is significant for their development and success. In the complex and volatile market environment, there are a high degree of risks and uncertainty for SEMs to carry out innovation activities[5]. Innovation consumes substantial cost and resources: Human, financial and material; Future is unpredictable, so it is too tough to judge whether the innovation is fruitful.

SEMs supply chain coordinative innovation is quite favorable for SEMs: firstly, SEMs own simple organizational structure and flexible management system, which help SEMs capture market demand information quickly so that they can combine innovation and market demand effectively. However, innovation requires investment in technology professionals, capital, Facilities and equipment and so on. By supply chain collaboration, SEMs can get support from other cooperative enterprises. In this way, supply chain realizes complementary resources, making full use of every dominant position as soon as possible and reducing waster to the minimum.

Secondly, the financing capacity of SEMs is inferior. Given this, SEMs are cautious on bearing risks. In some respects, SEMs' innovation may be related to funds, intellectual property rights, Laws and policies, which hinder innovation activity carry on, so that ongoing innovation behavior has no choice but stop. If this happens, innovative enterprises will suffer enormous losses, even go bankrupt. SEMs supply chain collaboration can largely reduce the loss of risks. Some Large companies in supply chain are able to raise funds, hire a legal expert or communicate with government officials if necessary. SEMs have more power in innovation if more firms are charged with the innovative risks.

\section{Threats and Temptations from Innovative Small and Medium-sized Enterprises}

We will introduce a simple model to explain why SEMs supply chain carry on collaboration in this part.

Consider a two-echelon supply chain that include two manufactures and two retailers (see Fig. 2 SME Supply Chain single model). We assume that the marketing price is fixed, and R1and R2 respectively 
represent the marketing price minus corresponding retailer's cost, $\mathrm{C} 1$ and $\mathrm{C} 2$ respectively represent corresponding manufacture's cost of production. As reference, Lovejoy [6] solve this problem, getting the transfer price:

$$
P=0.5 *\{(\mathrm{R} 1 \wedge \mathrm{C} 2)+(\mathrm{R} 2 \vee C 1)\}
$$

Then, the profits of retailers and manufactures are:

$$
\begin{array}{ll}
\pi_{r}^{i}=R_{i}-P, & (i=1,2) \\
\pi_{m}^{i}=P-C_{i}, & (i=1,2)
\end{array}
$$

Compared to large enterprises,SEMs are at a disadvantage in management and technology and they cost more in the process of procurement and production. Therefore, we assume $C 1 \leq C 2$, that is to say, the gap between large enterprises and SMEs is reflected in the cost. Here, $(a \vee b)=\max \{\mathrm{a}, \mathrm{b}\}$, $(a \wedge b)=\min \{\mathrm{a}, \mathrm{b}\}$.

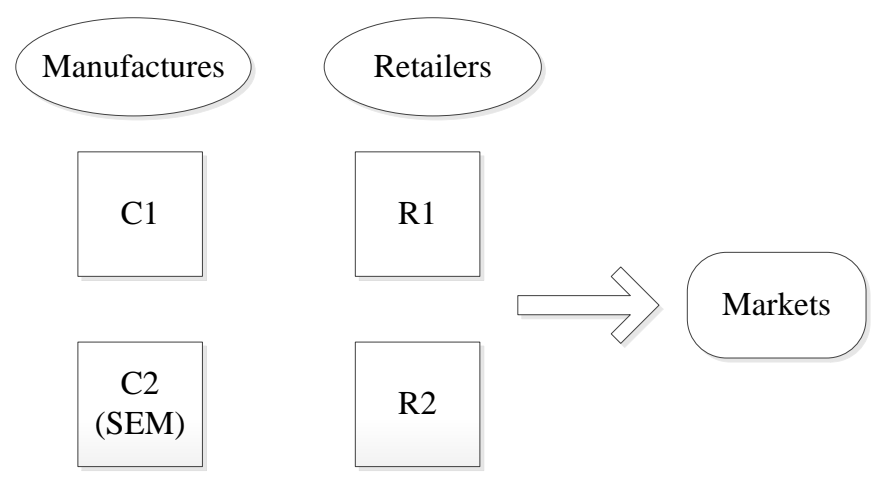

Fig. 2 SME Supply Chain single model

In this model, SEM will carry out innovative activities, which may reduce the cost of production if innovation is successful, that is, $\mathrm{C} 2$ drops. The changes after innovation are shown in Table 1 according

\begin{tabular}{|c|c|c|c|c|}
\hline \multicolumn{2}{|r|}{ Before Innovation } & \multicolumn{3}{|c|}{ After Innovation } \\
\hline & The transfer price & \multirow{4}{*}{$\mathrm{C} 2 \downarrow$} & The transfer price & Changes in profit \\
\hline$R 1 \geq C 2$ & $P=0.5 *\{\mathrm{C} 2+(\mathrm{R} 2 \vee C 1)\}$ & & $P=0.5^{*}\{\mathrm{C} 2+(\mathrm{R} 2 \vee C 1)\} \downarrow$ & $\pi_{r}^{i} \uparrow \quad \pi_{m}^{1} \downarrow$ \\
\hline \multirow{2}{*}{$R 1<C 2$} & \multirow{2}{*}{$P=0.5 *\{R 1+(\mathrm{R} 2 \vee C 1)\}$} & & $P=0.5^{*}\{R 1+(\mathrm{R} 2 \vee C 1)\} \leftrightarrow$ & $\pi_{r}^{i} \leftrightarrow \pi_{m}^{1} \leftrightarrow$ \\
\hline & & & $P=0.5^{*}\{\mathrm{C} 2+(\mathrm{R} 2 \vee C 1)\} \downarrow$ & $\pi_{r}^{i} \uparrow$ \\
\hline
\end{tabular}
to the equations above.

Table 1 The Changes Before Innovation and After Innovation

From Table 1, we can see that, the retailers' profits increase and competitive firms ' profits drop in some cases after SEM's innovation. Namely, SEMs in the supply chain taking innovation not only bring more 
profits for their down-stream buyers, but also make threats for their competitive enterprises. In order to avoid the reduction in profits, the competitive enterprises have the motive to make coordination with SEMs. As well, downstream firms may participate in coordination to keep profits increased.

\section{Conclusions}

As SEMs supply chain have enough justifications and motivations to make coordination, all the firms in the supply chain should make full use of their own advantages and resources to create more value for themselves, as well as the society.

A topic for our future research is how to make better collaboration in SEMs supply chain from the perspective of practice. At the same time, SEMs' survival and development are concerned with the stability of the society. So more work in this direction is needed.

\section{References}

[1] Gilder G. The revitalization of everything: the law of the microcosm[J]. Harvard Business Review. 1988, 66(2): 49-61.

[2] Freeman C, Soete L L. The economics of industrial innovation[M]. Psychology Press, 1997.

[3] Morrissey B, Pittaway L. A study of procurement behaviour in small firms[J]. Journal of Small Business and Enterprise Development. 2004, 11(2): 254-262.

[4] Wagner S M, Friedl G. Supplier switching decisions[J]. European Journal of Operational Research. 2007, 183(2): 700-717.

[5] Mohnen P, Röller L. Complementarities in innovation policy[J]. European Economic Review. 2005, 49(6): 1431-1450.

[6] Lovejoy W S. Bargaining chains[J]. Management Science. 2010, 56(12): 2282-2301. 